\title{
Exercise in the setting of hematopoietic stem cell transplantation
}

\author{
Joachim Wiskemann
}

Received: 5 December 2012 / Accepted: 8 December 2012 / Published online: 8 January 2013

(C) European Group for Research into Elderly and Physical Activity (EGREPA) 2013

\begin{abstract}
The procedure of hematopoietic stem cell transplantation (HSCT) is an intense treatment approach to cure patients from leukemia or lymphoma. Prior, during, and after HSCT, patients experience considerable physical and psychosocial distress. In light of the increasing number of successfully treated patients, the need is growing for evidence-based adjuvant therapy options, which are able to reduce treatment-related side effects and enhance the rehabilitation process. Exercise constitutes to be a promising intervention in this setting due to its multidimensional effectiveness. The purpose of this article was to review the current knowledge in the field of exercise and HSCT. Therefore, an electronic literature search in PubMed on the topic was performed. Twenty-nine articles could be identified and classified as relevant for this paper. Reviewed studies suggest that exercise training is an important therapeutic approach in the supportive care for transplant patients. Significant benefits from the exercise interventions have been reported for physical performance, quality of life (QoL), and fatigue status. Several other benefits, such as a more rapid immune recovery or alleviation of therapyrelated side effects have been reported in some studies. Future studies should address existing methodological problems, identify further effect levels of exercise and integrate a long-term perspective for the participants in the context of hematopoietic stem cell transplantation. Furthermore, potential biological pathways of exercise in HSCT patients should be investigated.
\end{abstract}

J. Wiskemann $(\bowtie)$

Working Group Physical Activity and Cancer, Divisions Medical

Oncology and Preventive Oncology, National Center for Tumor

Diseases (NCT), German Cancer Research Center (DKFZ)

University Clinic Heidelberg,

Im Neuenheimer Feld 460,

69120 Heidelberg, Germany

e-mail: joachim.wiskemann@nct-heidelberg.de
Keywords Exercise $\cdot$ Cancer $\cdot$ Stem cell transplantation $\cdot$ Review $\cdot$ Physical activity

\section{Introduction}

Hematopoietic stem cell transplantation (HSCT) is the only curative treatment for hematologic and lymphoid cancers to date but can also be used in the treatment of solid tumors and some non-malignant disorders [9]. HSCT varies according to the source of the substitute stem cells; depending on whether autologous stem cells (self-donor) or allogeneic stem cells (histologically compatible donor) are used for transplantation. Depending on the transplantation procedure, the duration of hospitalization ranges from 2 to 6 weeks provided that no major complications are incurred. Due to potential major complications, only patients in good physical conditions, with no or well-controlled co-morbidities up to an age of about 70 years, are usually eligible for HSCT.

Before, during, and after HSCT, patients experience substantial physical, psychological, and psychosocial distress and deteriorations in QoL. The prolonged treatment process leads to a decline in physical functioning due to the side effects of medical treatment $[25,28]$. Patients experience these stress symptoms even when the treatment is effective and they can be detrimental to the long-term success of HSCT. The most relevant treatment-related problems include:

- loss of physical performance/fitness

- fatigue

- distress and emotional problems (e.g., anxiety, depression)

- pain

- immunological/ hematological changes.

Additionally, common side effects such as GVHD, infections, diarrhea, serious oral mucositis, nausea, and pain $[3$, 
11] exacerbate the physical and psychological problems. Moreover, several other factors such as isolation during hospitalization, rapid and unsteady changes in the clinical status and the not unsubstantial risk of dying from the procedure are a strain on the patients and can increase the symptom burden.

In summation, all of these various physical and psychosocial stress factors and problems massively affect the individual QoL of each patient [1, 6, 27]. Moreover, QoL after transplantation is often adversely influenced by problems of reintegration into the community [30]. As described by Backer et al. [3], some of the affected persons are completely or partly unable to perform successfully in their previous social role functions. The treatment-related psychophysical problems described above also contribute to the reintegration difficulties. Furthermore, long-term survivors of HSCT are more likely to develop diabetes, cardiovascular diseases, osteoporosis, exercise-induced shortness of breath, and other ailments [2, 31].

At present, there is a growing interest in interventions which can help to alleviate these problems. Physical exercise therapy constitutes a potentially promising adjuvant intervention for HSCT patients because of its multidimensional effectiveness.

\section{Exercise intervention studies}

Until today, 29 publications in the field of exercise in patients prior to, during, or after HSCT have been published. These publications draw evidence from 23 separate clinical studies. A total of 1,177 patients with different hematological malignancies have been enrolled in these studies. The sample size of the individual studies varied from 12 to 135 patients. The majority of the participants $(721=61.3 \%)$ received allogeneic stem cell transplantation.

As a rule, exercise interventions were implemented during and/or after the hospitalization phase. Nine out of 23 studies evaluated the effects of endurance training, whereas only two studies investigated specific effects of isolated resistance training. The majority of studies $(n=12)$ investigated a combination of endurance and resistance training with one study including relaxation exercises as a third part of their intervention concept $[21,22]$.

\section{Outcomes}

Patients appeared to benefit from aerobic exercise regardless of whether the endurance training was performed in an inpatient, outpatient, or home-based setting. The three studies that focused on exercise in an inpatient setting $[4,5,14$, $21,33]$ showed that regular endurance training may not lead to an increase in endurance performance, but the loss of performance in the experimental groups was significantly lower than in control groups. Retrospective rehabilitation data analyzed by Morris et al. [29] even did not show improvements in endurance performance either, whereas a subgroup of $40 \%$ demonstrated improvement under glucocorticoid therapy. Non-randomized aerobic exercise intervention studies which started during the inpatient setting and were continued in the outpatient context, respectively, took place in the outpatient setting only, showed significantly enhanced endurance values in pre-/post-comparisons [7, 13, 18]. These results were supported by randomized controlled trials in the outpatient setting also showing substantial improvement in endurance performance compared to the control groups $[15,24,33]$.

Similar to the aerobic exercise interventions data, studies focusing on resistance training could only show stabilizing effects in strength performance, but no improvement, during the course of inpatient treatment. One study noted increased isometric muscle strength values for the lower body in the experimental group in their in-/outpatient trial but no significant effects for upper body muscles [33]. Comparable data could be shown by others $[4,16,26]$. However, a Danish researcher group showed significant effects in muscle strength between experimental and control group in relatively small allo-HSCT study population [21]. Another publication reported significant increases in strength values in their outpatient trial, also showing that the effect was maintained up to a 3-month follow-up [24].

Regarding body weight and composition, two studies $[8,17]$ showed increases after regular resistance and endurance training. In the study of Coleman et al. [8], fat-free mass increased in the exercise group, whereas the controls lost weight during the same time period. In the study performed by Hayes et al. [17], the experimental group evidenced a reduced percentage of body fat, an enhanced fat-free mass and a significant increase in total energy expenditure compared with pre-treatment measurements. Cunningham et al. [10] on the other hand, did not find any significant changes between body composition values in patients.

In case of immunological changes, the study done by Dimeo et al. [14] showed that patients who performed a daily endurance training had a significantly shorter duration of neutropenia in comparison to the non-exercising controls. Additionally, there was a trend toward shorter duration of thrombopenia. Kim et al. [23] reported a significant improvement in the lymphocyte count in patients who performed a daily 30-min exercise program in bed for 6 weeks. Lymphocyte counts among controls decreased significantly, whereas the values of the exercisers remained steady. In contrast to those findings, another study, who examined the number and function of lymphocytes and leukocytes, did not observe any significant differences 
between the experimental and control groups at the end of the intervention [20].

Most exercise studies among HSCT patients investigated several dimensions of quality of life (QoL) including physical function and emotional/distressing aspects, severity of treatment- and/or disease-related side effects as well as overall QoL scores. The studies mostly demonstrated improvements regarding overall QoL and physical functioning in exercisers versus deteriorations among controls [5, 12, 19, 24, 32, 33].

As discussed above, fatigue is a common and serious side effect in HSCT. Several studies showed that the patient's status of fatigue can be positively altered by exercise. Randomized controlled data from our working group [33] support these findings, revealing a significant better development of fatigue symptoms in the experimental group (up to $+15 \%$ ) compared to controls (up to $-28 \%$ ). A study done by Carlson et al. [7] also showed highly significant intervention effects on fatigue after a 12 -week outpatient treatment. These improvements in fatigue were almost completely maintained over the 1-year follow-up measurement period. Wilson et al. [32] noted a reduction of severity of fatigue symptoms due to their aerobic intervention program in the home-based setting more than 6 month after HSCT. Similarly, Hacker et al. [16] showed significant improvements in fatigue through resistance training.

Only a few studies reported on results of treatmentrelated complications such as severe pain, diarrhea, mucositis, cognitive problems, infections, and duration of hospitalization. In one study, the experimental group showed in comparison to the control group significantly better results for the parameters severity of diarrhea, duration of hospitalization, and severity of pain at time of discharge [14]. The results concerning the severity of pain were supported by the authors' observation that $25 \%$ of the patients in the experimental group, in contrast to only $3 \%$ in the control group, did not need analgesia during hospitalization. Another study evaluated data on five symptom clusters: (a) mucositis, (b) cognitive, (c) gastrointestinal, (d) affective, and (e) functional cluster. The experimental group showed significant reduction in the severity of the symptoms in all clusters except for those subsumed under the affective cluster (d) [22]. Furthermore, the study done by Knols et al. showed in their outpatient intervention a significant difference between both groups in terms of severity of diarrhea [24].

\section{Summary and conclusions}

To date, 23 reports of the effects exercise interventions have been published in the field of hematopoietic stem cell transplantation. Fourteen of them were randomized controlled trials. Two studies evaluated retrospective data. The majority of studies $(n=12)$ evaluated a combination of endurance and resistance training. Due to the heterogeneity of the intervention strategies and assessment methods used, it is difficult to make direct comparison and to draw clear conclusions. In spite of these limitations, the results reported from randomized controlled trials indicate that endurance training/aerobic exercise or resistance training can induce beneficial physical adaptation processes and can help to alleviate complications and side effects (e.g., fatigue, QoL, and distress) for patients under allogeneic as well as autologous HSCT treatment. Patients tend to be highly motivated and adhere to the interventions. No serious adverse events have been reported to date. Studies also suggests that there might be an impact on immune function and recovery.

Despite the promising findings, various methodological problems (e.g., small sample sizes or uncontrolled design) need to be addressed in future research. The following goals should be pursued: (1) validation of the up-to-date reported positive results on physical performance, QoL, and treatment-related side effects; (2) investigation of major clinical endpoints, including length of hospitalization, recurrence rates, GVHD, and survival; (3) identification and investigation on underlying biological mechanisms; and (4) assessing exactly the dose-response so that precise and well-aimed exercise recommendations can be made.

Future research needs also to give more consideration to the different hematological entities, to distinguish more between autologous and allogeneic treatment procedures and different conditioning regimens of HSCT because differing treatment modalities and disease type have a varied influence on patients' initial condition and have a strong impact on the prognosis and frequency and severity of side effects.

Conflict of interest The author declares no conflict of interest.

\section{References}

1. Andrykowski MA, Bishop MM, Hahn EA et al (2005) Long-term health-related quality of life, growth, and spiritual well-being after hematopoietic stem-cell transplantation. J Clin Oncol 23(3):599 608

2. Baker KS, Ness KK, Weisdorf D et al (2010) Late effects in survivors of acute leukemia treated with hematopoietic cell transplantation: a report from the Bone Marrow Transplant Survivor Study. Leukemia 24(12):2039-2047

3. Baker F, Zabora J, Polland A, Wingard J (1999) Reintegration after bone marrow transplantation. Cancer Pract 7(4):190-197

4. Baumann FT, Kraut L, Schule K, Bloch W, Fauser AA (2010) A controlled randomized study examining the effects of exercise therapy on patients undergoing haematopoietic stem cell transplantation. Bone Marrow Transplant 45(2):355-362

5. Baumann FT, Zopf EM, Nykamp E et al (2011) Physical activity for patients undergoing an allogeneic hematopoietic stem cell 
transplantation: benefits of a moderate exercise intervention. Eur $\mathrm{J}$ Haematol 87(2):148-156

6. Bieri S, Roosnek E, Helg C et al (2008) Quality of life and social integration after allogeneic hematopoietic SCT. Bone Marrow Transplant 42(12):819-827

7. Carlson LE, Smith D, Russell J, Fibich C, Whittaker T (2006) Individualized exercise program for the treatment of severe fatigue in patients after allogeneic hematopoietic stem-cell transplant: a pilot study. Bone Marrow Transplant 37(10):945-954

8. Coleman EA, Coon S, Hall-Barrow J, Richards K, Gaylor D, Stewart B (2003) Feasibility of exercise during treatment for multiple myeloma. Cancer Nurs 26(5):410-419

9. Copelan EA (2006) Hematopoietic stem-cell transplantation. N Engl J Med 354(17):1813-1826

10. Cunningham BA, Morris G, Cheney CL, Buergel N, Aker SN, Lenssen P (1986) Effects of resistive exercise on skeletal muscle in marrow transplant recipients receiving total parenteral nutrition. JPEN J Parenter Enteral Nutr 10(6):558-563

11. Danaher EH, Ferrans C, Verlen E et al (2006) Fatigue and physical activity in patients undergoing hematopoietic stem cell transplant. Oncol Nurs Forum 33(3):614-624

12. DeFor T, Burns L, Gold E, Weisdorf D (2007) A randomized trial of the effekt of a walking regimen on the functional status of 100 adult allogeneic donor hematopoietic cell transplant patients. Biol Blood Marroww Transplant 13:948-955

13. Dimeo F, Bertz H, Finke J, Fetscher S, Mertelsmann R, Keul J (1996) An aerobic exercise program for patients with haematological malignancies after bone marrow transplantation. Bone Marrow Transplant 18(6):1157-1160

14. Dimeo F, Fetscher S, Lange W, Mertelsmann R, Keul J (1997) Effects of aerobic exercise on the physical performance and incidence of treatment-related complications after high-dose chemotherapy. Blood 90(9):3390-3394

15. Dimeo FC, Tilmann MH, Bertz H, Kanz L, Mertelsmann R, Keul J (1997) Aerobic exercise in the rehabilitation of cancer patients after high dose chemotherapy and autologous peripheral stem cell transplantation. Cancer 79(9):1717-1722

16. Hacker ED, Larson J, Kujath A, Peace D, Rondelli D, Gaston L (2010) Strength training following hematopoietic stem cell transplantation. Cancer Nurs 34(3):238-249. doi:10.1097/NCC.0b013e3181fb3686

17. Hayes S, Davies PS, Parker T, Bashford J (2003) Total energy expenditure and body composition changes following peripheral blood stem cell transplantation and participation in an exercise programme. Bone Marrow Transplant 31(5):331-338

18. Hayes SC, Davies PS, Parker TW, Bashford J, Green A (2004) Role of a mixed type, moderate intensity exercise programme after peripheral blood stem cell transplantation. Br J Sports Med 38 (3):304-309

19. Hayes S, Davies PS, Parker T, Bashford J, Newman B (2004) Quality of life changes following peripheral blood stem cell transplantation and participation in a mixed-type, moderateintensity, exercise program. Bone Marrow Transplant 33 (5):553-558
20. Hayes SC, Rowbottom D, Davies PS, Parker TW, Bashford J (2003) Immunological changes after cancer treatment and participation in an exercise program. Med Sci Sports Exerc 35(1):2-9

21. Jarden M, Baadsgaard MT, Hovgaard DJ, Boesen E, Adamsen L (2009) A randomized trial on the effect of a multimodal intervention on physical capacity, functional performance and quality of life in adult patients undergoing allogeneic SCT. Bone Marrow Transplant 43(9):725-737

22. Jarden M, Nelausen K, Hovgaard D, Boesen E, Adamsen L (2009) The effect of a multimodal intervention on treatment-related symptoms in patients undergoing hematopoietic stem cell transplantation: a randomized controlled trial. J Pain Symptom Manage 38 (2):174-190

23. Kim SD, Kim HS (2006) A series of bed exercises to improve lymphocyte count in allogeneic bone marrow transplantation patients. Eur J Cancer Care (Engl) 15(5):453-457

24. Knols RH, de Bruin ED, Uebelhart D et al (2011) Effects of an outpatient physical exercise program on hematopoietic stem-cell transplantation recipients: a randomized clinical trial. Bone Marrow Transplant 46(9):1245-1255

25. Kovalszki A, Schumaker GL, Klein A, Terrin N, White AC (2008) Reduced respiratory and skeletal muscle strength in survivors of sibling or unrelated donor hematopoietic stem cell transplantation. Bone Marrow Transplant 41(11):965-969

26. Mello M, Tanaka C, Dulley FL (2003) Effects of an exercise program on muscle performance in patients undergoing allogeneic bone marrow transplantation. Bone Marrow Transplant 32(7):723-728

27. Mock V, Pickett M, Ropka ME et al (2001) Fatigue and quality of life outcomes of exercise during cancer treatment. Cancer Pract 9 (3):119-127

28. Morishita S, Kaida K, Ikegame K et al (2011) Impaired physiological function and health-related QOL in patients before hematopoietic stem-cell transplantation. Support Care Cancer 20 (4):821-829. doi:10.1007/s00520-011-1156-2

29. Morris GS, Brueilly KE, Scheetz JS, de Lima MJ (2011) Adherence of stem cell transplant recipients receiving glucocorticoid therapy to an exercise-based rehabilitation program. Support Care Cancer 20(10):2391-2398. doi:10.1007/s00520-011-1352-0

30. Pidala J, Anasetti C, Jim H (2009) Health-related quality of life following haematopoietic cell transplantation: patient education, evaluation and intervention. Br J Haematol 148(3):373-385

31. Tichelli A, Passweg J, Wojcik D et al (2008) Late cardiovascular events after allogeneic hematopoietic stem cell transplantation: a retrospective multicenter study of the Late Effects Working Party of the European Group for Blood and Marrow Transplantation. Haematologica 93(8):1203-1210

32. Wilson RW, Jacobsen PB, Fields KK (2005) Pilot study of a homebased aerobic exercise program for sedentary cancer survivors treated with hematopoietic stem cell transplantation. Bone Marrow Transplant 35(7):721-727

33. Wiskemann J, Dreger P, Schwerdtfeger R et al (2011) Effects of a partly self-administered exercise program before, during, and after allogeneic stem cell transplantation. Blood 117(9):2604-2613 